\title{
Investigation of Silicon Carbide Diode Structures via Numerical Simulations Including Anisotropic Effects
}

\author{
E.Velmre ${ }^{a}$, A.Udal $^{a}$, F.Masszi $^{\mathrm{b}}$ and E.Nordlander ${ }^{\mathrm{b}, \mathrm{c}}$ \\ a Tallinn Technical University, Inst. of Electronics, \\ Akadeemia tee 1, EE-0026 Tallinn,ESTONIA, evelmre@le.ttu.ee, audal@le.ttu.ee \\ b Scanner Lab, Electronics Dept., Inst. of Technology, Uppsala University, \\ P.O.Box 534, S-75121 Uppsala, SWEDEN, ferenc@sim.teknikum.uu.se \\ c Univ. College of Gävle-Sandviken, Dept. of Technology, \\ P.O.Box 6052, S-80006 Gävle, SWEDEN, enr@hgs.se
}

\begin{abstract}
The Poisson's and continuity equations based SiC-DYNAMIT-1DT/2DT simulators with anisotropic material analysis capabilities were developed. A comparison of experimental and simulated forward $\mathrm{I} / \mathrm{V}$ curves for three $6 \mathrm{H}-\mathrm{SiC} \mathrm{P}^{+} \mathrm{NN}^{+}$diodes is presented and the related model parameter adjustment problems are discussed. The influence of the strong electron mobility anisotropy on carrier distributions is investigated and the existence of a "mobility anisotropy induced anomalous charge accumulation effect" is demonstrated.
\end{abstract}

\section{Introduction}

The progress in $\mathrm{SiC}$ device technology is enhancing the development of the simulators in order to link material data to the electrical behaviour of devices. Recently, a $4.5 \mathrm{kV} 6 \mathrm{H}$ $\mathrm{SiC}$ diode has been reported [1] with acceptable electrical performance. However, in this work, like in others, e.g. [2,3] the measured results, particularly the high forward current voltage drops, disagree with simulated results. This emphasises the need of a correct physical description, i.e. first of all the development of temperature and doping-dependent lifetime and mobility models. Another problem for simulator development is the material anisotropy, especially related to $6 \mathrm{H}-\mathrm{SiC}$ (power applications), and less to $4 \mathrm{H}-\mathrm{SiC}$ (highfrequency applications). In the following, a remarkable influence of the strong electron mobility anisotropy on the forward-biased pn-junction behaviour is demonstrated.

In connection with the present work, the $1 \mathrm{D}$ and $2 \mathrm{D}$ nonisothermal simulators SiCDYNAMIT-1DT/2DT, developed at Tallinn Technical University, were also tested against the recent version of MEDICI [4] with anisotropic material simulation capabilities (but without any physical default data). If the same input data and mesh were carefully specified for forward-biased tasks like the ones described below, then a fair coincidence of results was obtained.

\section{Comparison of experimental and simulated forward $I / V$ characteristics}

In the present $1 \mathrm{D}$-simulations the $\mathrm{n}_{i}(\mathrm{~T})[5], \mu_{n} \|, \mu_{\mathrm{p}}\left(\mathrm{N}_{\mathrm{D}}+\mathrm{N}_{\mathrm{A}}, \mathrm{T}\right)[6], \varepsilon_{\|}=10.03$ [7] ("I denotes "parallel to c-axis") and the conventional single-level SRH recombination model were used. The doping-dependent bandgap narrowing, Auger recombination, electronhole scattering, mobility field-dependence and incomplete impurity ionization models were omitted, mainly due to the lack of reliable data. The impact ionization [5] was not applied either in the present forward-biased simulations. Fig. 1 shows results for a realistic $6 \mathrm{H}-\mathrm{SiC}$ diode structure [3]. 


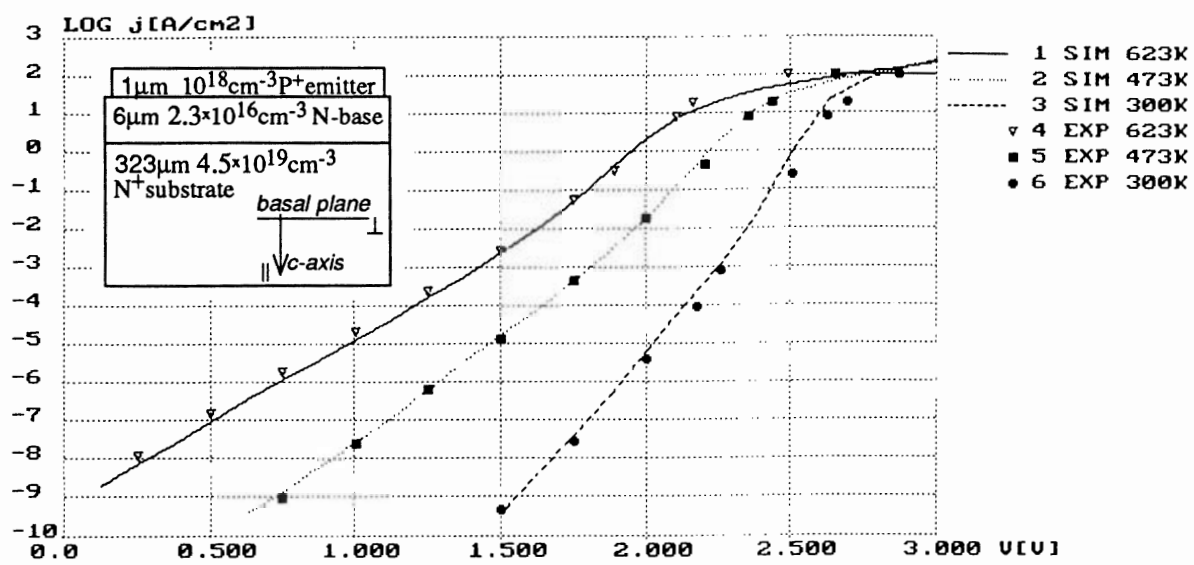

Fig.1 Simulated and experimental forward I/V characteristics at 3 temperatures

To achieve a better fit in Fig.1, the mobility temperature-dependence exponent value of -1.8 was added to the Caughey-Thomas like doping-dependence formulae from a recent work of Cree Corp. researchers [6] (authors of [6] suggest values -2.07...-1.8). Greater exponent absolute values would too strongly decrease mobilities at higher temperatures, and it would cause the $\mathrm{I} / \mathrm{V}$ curves crossing point to move down to current densities not matching present experiments. For SRH capture times the values $\tau_{\mathrm{po}}(300 \mathrm{~K})=$ $\tau_{\text {no }}(300 \mathrm{~K})=0.2 \mathrm{~ns}$ (emitters) and $2 \mathrm{~ns}$ (base) were used together with the temperaturedependence exponent of 1.5 (applied only for the base region). However, it should be pointed out, that to achieve better fit, considerably lower lifetime values have been used here in comparison with the 100ns range lifetimes observed in [1].

Fig.2 gives comparison results for two analogous diode structures, for which the matching of simulation and experiment is quite poor. These $4.5 \mathrm{kV}$ and $2.2 \mathrm{kV}$ structures were specified according to refs. [1] and [2], respectively. In the simulations $\tau_{\mathrm{po}}=\tau_{\mathrm{no}}=1 \mathrm{~ns}$ was taken for all regions, except $\mathrm{N}$-bases where $\tau_{\mathrm{po}}=430 \mathrm{~ns}[1](4.5 \mathrm{kV}$ device$)$ and $\tau_{\mathrm{po}}=100 \mathrm{~ns}$ $(2.2 \mathrm{kV}$ device) were set.
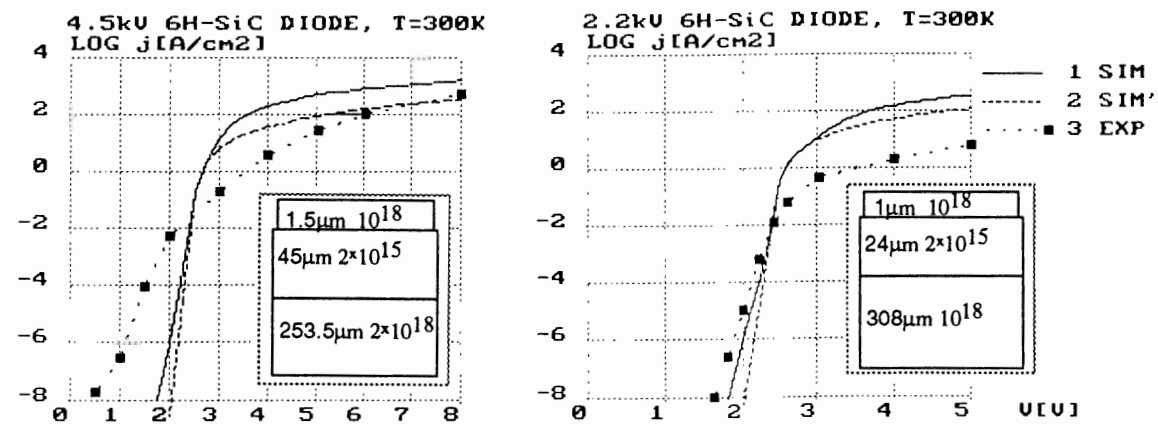

Fig.2 Some additional comparison results for two high-voltage diode structures

Main discrepancies between simulation and experiment can be observed at higher current densities, where simulated voltage drops remain remarkably lower than experimental ones. This may be due to additional contact or probe resistances in experiments, but, for instance, can be explained also as a result of declining lifetime injection-dependence caused by non-midgap recombination centers. The dashed lines in Fig. 2 correspond to simulations, where the $S R H$ recombination model parameters $n_{1}, p_{1}$ were specially chosen to achieve a 10-fold lifetime decrease at high injection. Mismatch in the low-current region of the $4.5 \mathrm{kV}$ device may be caused by device self-heating during the experiments. 


\section{Two-dimensional simulations with anisotropic effects}

To investigate the anisotropy influence, a special $16 \mu \mathrm{m}^{\times} 16 \mu \mathrm{m} 2 \mathrm{D}$-structure was constructed (see Fig.4). The doping profile of this abrupt-junction diode has been taken by the diode from Fig. 1 (but including only $1 \mu \mathrm{m}$ of the thick $\mathrm{N}^{+}$substrate). Furthermore, it has a total geometrical symmetry with respect to the $\mathrm{x}=\mathrm{y}$ line. The right and bottom contacts were grounded and the voltage was applied to the $\mathrm{P}^{+}$emitter contact in the upper left part of structure. The dimensions were selected so that $1 \mathrm{~A}$ current corresponds to an average current density $\approx 1 \mathrm{~A} / \mathrm{cm}^{2}$ for all electrodes. Thus any anisotropy influence could clearly be observed as a difference between the right and bottom contact currents or as a symmetry distortion in physical quantity distributions.

While not paying attention to breakdown operation modes (strong impact ionization anisotropy) and nonisothermal heat transfer problems (heat conductivity anisotropy $\approx 0.7$ for $6 \mathrm{H}-\mathrm{SiC}$ ), it can be stated for $6 \mathrm{H}-\mathrm{SiC}$, that beside the low anisotropy of the dielectric permittivity $\varepsilon \perp / \varepsilon \|=9.66 / 10.03$ [7], the $\mu_{n}$ anisotropy $\mu_{n} \perp / \mu_{n} \|=4.8$ is already a remarkable number and it stays quite constant over a wide temperature and doping range [6]. Reverse-biased simulations show that the weak $4 \% \varepsilon$ anisotropy causes only $\approx 2 \%$ difference in the field penetration depth and maximum field, following a square root law.

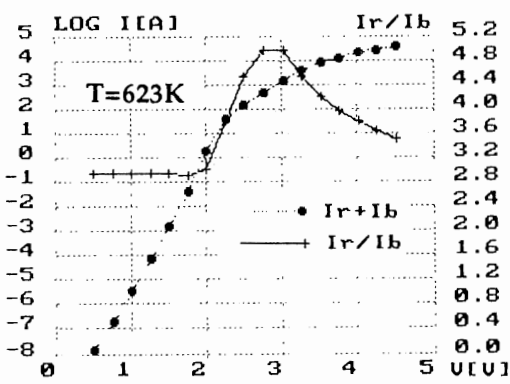

Figs.3,4 present the main results of the forwardbiased simulations to investigate the mobility anisotropy influence. The ratio of horizontal (i.e. direction with greater mobility) to vertical current moves from a constant level at low currents to the peak at medium currents. At high currents, the transition to a more uniform current distribution may be observed again (see also the flowlines in Fig.4). As numerical experiments show, these changes are defined both by mobility anisotropy and structure geometry.

Fig. 3 Right and bottom contact current sum and ratio versus forward bias
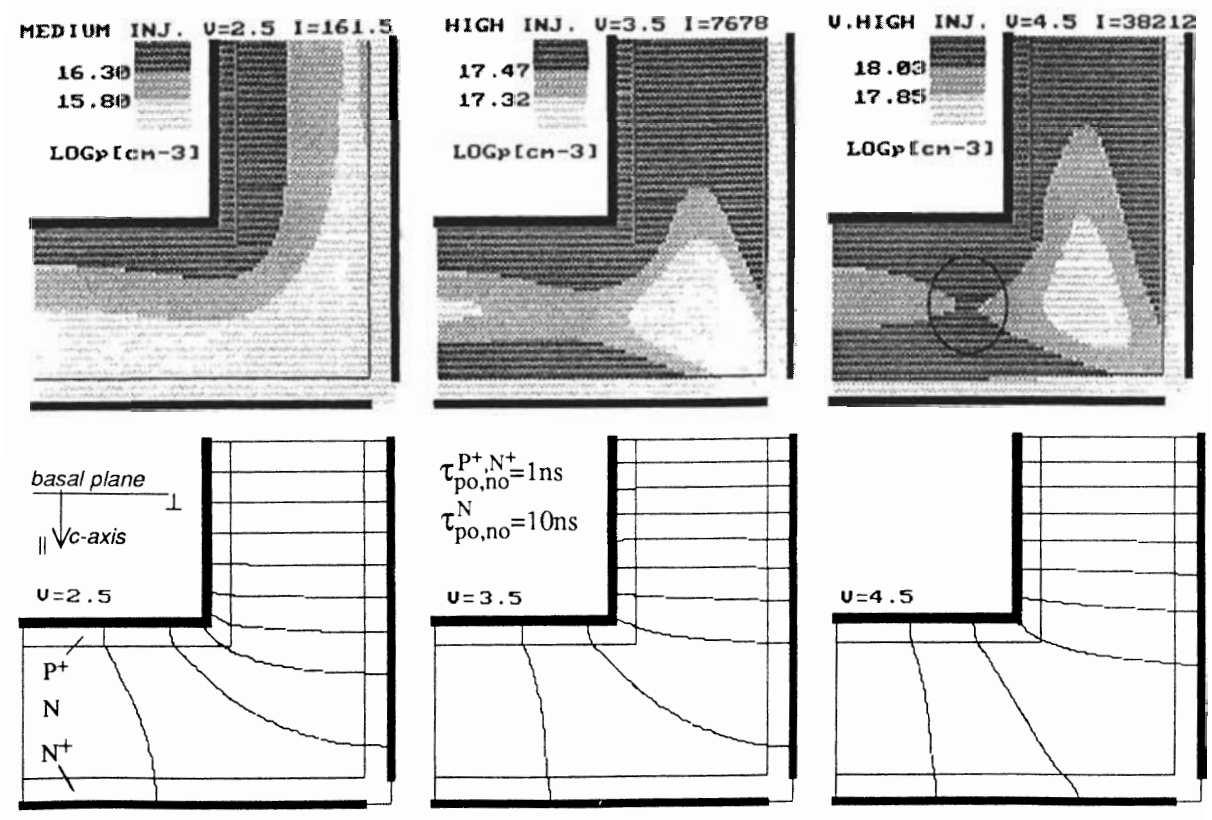

Fig.4 Hole density and total current flowline maps at three forward bias 


\section{The anomalous charge accumulation effect}

Fig.4 shows that the "normal" $\mathrm{p}(\mathrm{x}, \mathrm{y})$ distributions (where higher carrier density corresponds to higher mobility, like in the quasi-1D-regions near to the top and left boundaries of the structure) obtain an anomalous character by the transition to high injection mode near the pn-junction corner. An explanation for this "mobility anisotropy induced anomalous charge accumulation" is given on the basis of pn-junction and base voltage drops balance analysis in Fig.5. This theory assumes high injection in the N-base, spatially constant hole densities $\mathrm{p}_{\|}, \mathrm{p}_{\perp}$ and as a quite rough estimation, the equality of vertical and horizontal current densities. The last assumption is confirmed by flowline maps in Fig. 4 and by the fact that the considered area dimensions are of the same range as the charge carrier diffusion lengths. Compared to the predicted $\mathrm{p}_{\|} / \mathrm{p}_{\perp}=2.8$, Fig. 4 gives a value $\approx 1.5$. This can be explained by the inequality of vertical and horizontal current densities (greater horizontal current is equivalent to smaller $A$ in formulae of Fig.5).

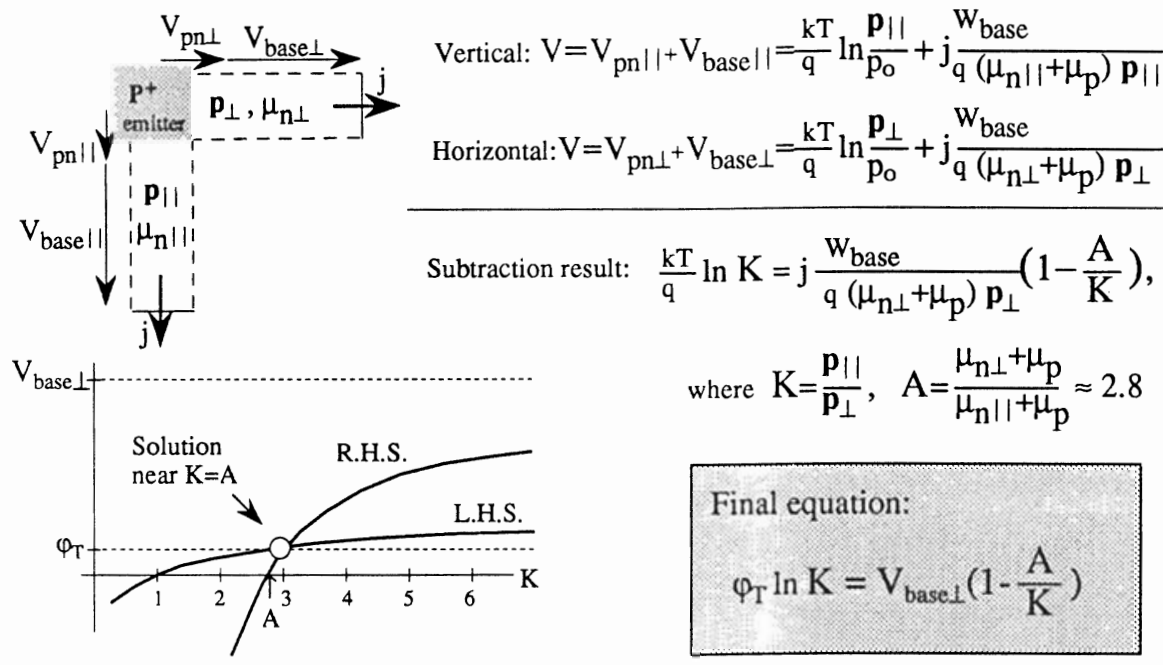

Fig.5 Theoretical explanation of the anomalous charge accumulation effect

\section{Acknowledgement}

This work has been supported by the Estonian Science Foundation (Project No.568), The Royal Swedish Academy of Sciences (Contract No.1489), the Swedish Institute and the Swedish National Board for Industrial and Technical Development (NUTEK Project P2305-1).

\section{References}

[1] O.Kordina, "Growth and Characterization of Silicon Carbide Power Device Material", Ph.D.Thesis, Linköping Studies in Science and Techn. Dissertation No.352, Linköping University, Sweden, 1994.

[2] P.G.Neudeck, D.J.Larkin, J.A.Powell and L.Matus, "2000V 6H-SiC p-n junction diodes grown by chemical vapour deposition", Appl.Phys.Lett. 64(11), 14 March 1994, pp.1386-1388.

[3] J.A.Edmond, H.-S. Kong and C.H. Carter Jr., "Blue LEDs, UV photodiodes and high-temperature rectifiers in 6H-SiC", Physica, v.B185, 1993, pp.453-460.

[4] TMA MEDICI, Two-Dimensional Device Simulation Program, Version 2.0 (including the Anisotropic Material Advanced Application Module), Sept. 1994, TMA Inc., Palo Alto, USA.

[5] E.Velmre and A.Udal, "Numerical Simulation of a Silicon Carbide Diode", Proc. of the 4th Biennial Conf. (Baltic Electronics Conf. 1994), Tallinn(Estonia), Oct.9-14, 1994, pp.559-566.

[6] Schaffer W.J., Negley G.H., Irvine K.G.and Palmour J.W., "Conductivity anisotropy in epitaxial 6H and 4H SiC", MRS Spring Meeting, San Francisco, Apr.4-8, 1994, 6 p.

[7] L.Patrick and W.J. Choyke, "Static Dielectric Constant of SiC", Phys.Rev., v.B2, 1970, pp.2255-2256. 